\title{
Neuro-Fuzzy Identification of an Internal Combustion Engine
}

\author{
Tengku N. A. Tuan Kamaruddin \\ Department of Mechanical Engineering \\ International Islamic University Malaysia \\ Kuala Lumpur, Malaysia \\ yana_akma@yahoo.com
}

\author{
Intan Z. Mat Darus \\ Faculty of Mechanical Engineering \\ UniversitiTeknologi Malaysia \\ Johor Bahru, Malaysia \\ intan@fkm.utm.my
}

\begin{abstract}
Dynamic modeling and identification of an internal combustion engine (ICE) model is presented in this paper. Initially, an analytical model of an internal combustion engine simulated within SIMULINK environment is excited by pseudorandom binary sequence (PRBS) input. This random signals input is chosen to excite the dynamic behavior of the system over a large range of frequencies. The input and output data obtained from the simulation of the analytical model is used for the identification of the system. Next, a parametric modeling of the internal combustion engine using recursive least squares (RLS) technique within an auto-regressive external input (ARX) model structure and a nonparametric modeling using neuro-fuzzy modeling (ANFIS) approach are introduced. Both parametric and nonparametric models verified using one-step-ahead (OSA) prediction, mean squares error (MSE) between actual and predicted output and correlation tests. Although both methods are capable to represent the dynamic of the system very well, it is demonstrated that ANFIS gives better prediction results than RLS in terms of mean squares error achieved between the actual and predicted signals.
\end{abstract}

Keywords - ANFIS, internal combustion engine, neuro-fuzzy modeling, recursive least square, system identification

\section{INTRODUCTION}

Dynamic modeling and identification of a system is a favored method to obtain the transfer function of the system in order to avoid dealing with the complexity of the systems and massive equations involved in constructing the engine model. It is a system's parameters estimation process of developing the mathematical model based on the input and output relation of the system [1]. It is very convenient to have the mathematical model representing the system and able to estimate the variables such as engine torque and speed particularly for highly nonlinear and time-varying characteristic such as the engine control system.

The procedure of system identification includes the data acquisition which usually acquired during experimental work. Then, the critical part is the selection of the model structure where understanding of the system is usually required. The main part of the identification is estimation of the model parameters [2-3]. There are many methods in estimating the behavior of a system. Least square, recursive least squares are good examples of known conventional methods. Other intelligent methods such as genetic algorithm, neural network are becoming more popular in terms of their application in system identification. Mean square error and correlation tests are the common methods used in validating the model. This paper emphasized on the implementation of RLS and ANFIS algorithm in system identification of an internal combustion engine.

\section{BACKGROUND STUDY}

The unknown parameters of a system are best to be determined during real time operations. One of the adaptive algorithms that is able to predict the parameters based on some known parameters and previous history is recursive least square (RLS). The main advantage of RLS is that it can continuously tune and update the estimated parameters during the operation [3].

The main design parameter in RLS, $\lambda$ is called forgetting factor. The value of $\lambda$ is defined between $0<\lambda$ $<1$. The function is to ensure the data in the past are forgotten in order to get the statistical variations of observable data when the filter operates in non-stationary environment [4-6]. Based on literature study, the value is chosen between the interval of 0.85-0.95 [7]. Other literature stated [8] that the usual value chosen is 0.9 to 0.99 .

For fuzzy logic, it replicates the way of human thinking and perception in making a decision of an automatic control strategy. It is applied widely in many applications because it is able to handle imprecise and uncertainty data despite the complexity of the processes. However, the disadvantage of fuzzy is the difficulty of choice and design of membership function to go well with a given problem. This can be overcome by the neural networks capability in term of learning, adaptation, identification of a system which could characterize a system based on the input output data. The integration of both is called adaptive neuro-fuzzy inference system (ANFIS) [9].

\section{PROPOSED IDEA}

Conventionally, mathematical modeling or nonlinear analytical model will be developed in order to apply the controller design of ICE and to compare with the performance data via experiment. But by applying the identification method, more accurate model representation 
could be obtained and the complicated formulation involved could be eliminated.

The ICE model used (Fig. 1) is referred to the Modelling Engine Timing Using Triggered Subsystems from SIMULINK Matlab [10]. It is based on Crossley and Cook [11] simulation results which was validated against dynamometer test data. The model presents a fourcylinder spark-ignition engine with throttle angle as input and engine speed as output.

The engine model is divided into five main elements: a) Throttle

$$
\begin{aligned}
& h_{a l}=f(\theta) g\left(R_{m}\right)=g g^{-1}
\end{aligned}
$$

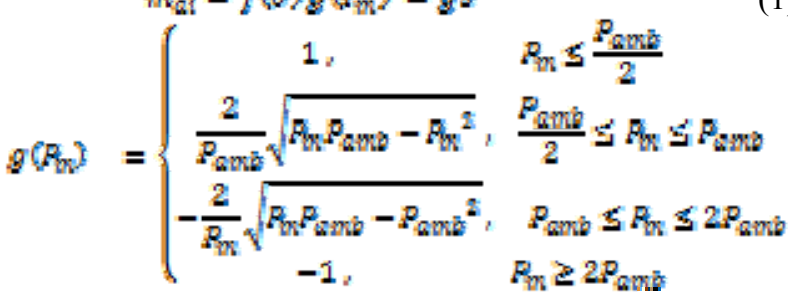

b) Intake manifold

$$
F_{\mathrm{w}}=\frac{\pi T}{\mathrm{I}_{\mathrm{m}}}\left(n_{\mathrm{al}}-n_{\mathrm{m}}^{\mathrm{k}}\right)
$$

where

$R \quad=$ specific gas constant

$T \quad=$ temperature $\left({ }^{\circ} \mathrm{K}\right)$

$V_{\mathrm{m}} \quad=$ manifold volume $\left(\mathrm{m}^{3}\right)$

$n_{\text {We }} \quad=$ mass flow rate of air out of manifold

$P_{m} \quad=$ rate of change of manifold pressure (bar/s)

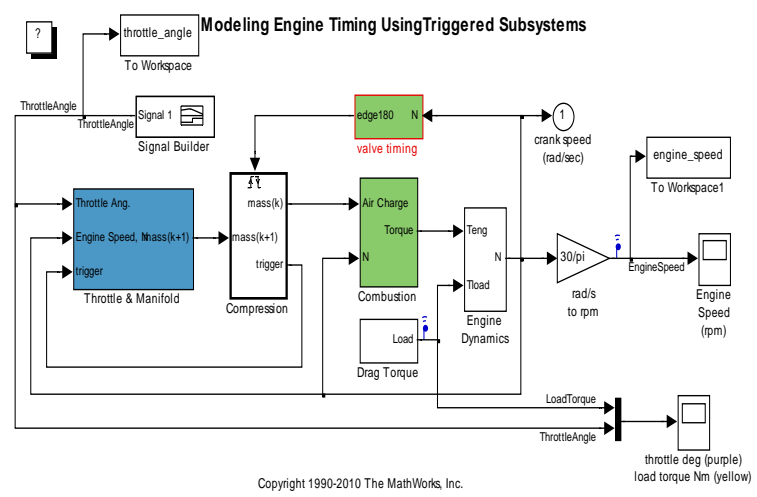

Figure 1. Modeling engine timing using triggered subsystems [10] c) Mass flow rate

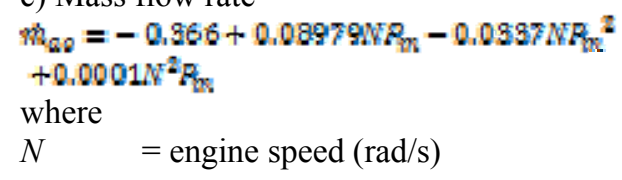

d) Compression stroke

This model has the intake, compression, combustion and exhaust strokes simultaneously which is at any given time, one cylinder is in each phase. Thus, the combustion of each intake charge is delayed by $180^{\circ}$ of crank rotation from end of the intake stroke.

e) Torque generation and acceleration

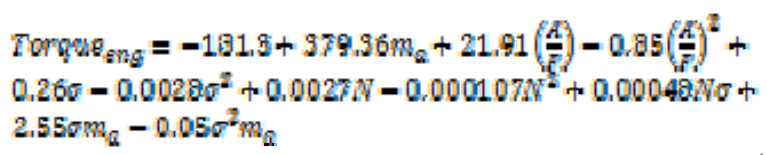

where

$$
\begin{aligned}
& m_{a} \quad=\text { mass of air in cylinder for combustion (g) } \\
& A / F \quad=\text { air fuel ratio } \\
& \sigma \quad=\text { spark advance (degree before TDC) } \\
& \text { Torque }_{\text {eng }}=\text { torque produced by the engine }(\mathrm{Nm}) \\
& \not \hat{V}=\text { Torque }_{\mathrm{eng}}-\text { Torqwe }_{\text {la }} \\
& \text { where } \\
& J \quad=\text { engine rotational moment of inertia }\left(\mathrm{kg}-\mathrm{m}^{2}\right) \\
& N \quad=\text { engine acceleration }\left(\mathrm{rad} / \mathrm{s}^{2}\right)
\end{aligned}
$$

\section{METHODOLOGY}

An input signal is applied to the internal combustion engine (ICE) model and the response is measured. The input signal needs to be sufficiently exciting and the data contains information of the system in a large range of frequencies. Pseudo-random binary signal (PRBS) is chosen as the input signal (Fig.2). It is called pseudorandom as it exhibits some properties that approximate a random signal [12]. From the data collected of 33707 data (Fig.3), 20000 data are chosen to be used. The first 10000 data are eliminated to make sure the system already in stable condition after excited by the input. The data is then normalized within range of -1 to 1.10000 data is used as the training data and another 10000 data is used for checking/validation. 


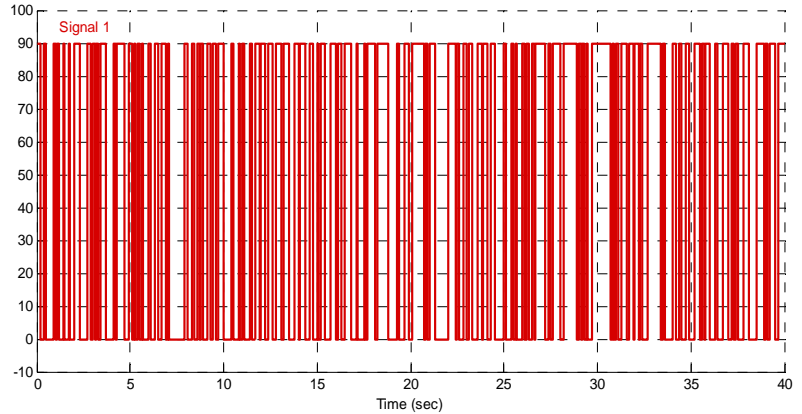

Figure 2. Pseudorandom input signal

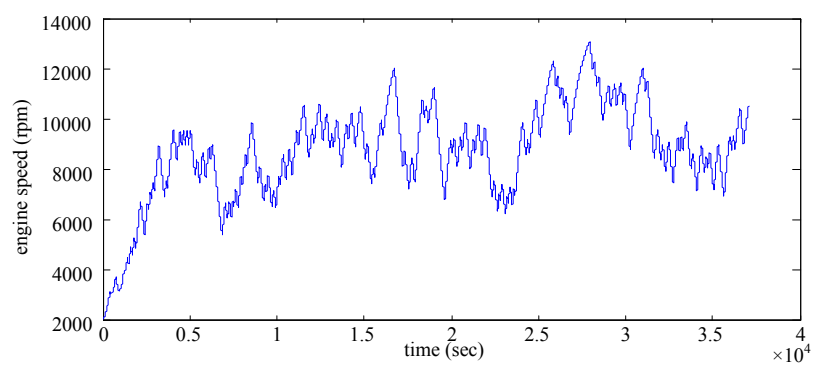

Figure3. Engine speed data (output signal)

\section{A. Model Structure Selection}

Model structure selection is very important to determine the possible solutions of the system [13]. This step is important but yet difficult choice to be made. In this research, the simplest form of input-output polynomial model of a linear ARX model structure is chosen. The system can be represented as:

$y(t)=\frac{B\left(z^{-1}\right)}{A\left(z^{-1)}\right.} u(t)+\frac{k(0)}{A\left(z^{-1)}\right.}$

where

$A\left(z^{-1}\right)=1+a_{1} z^{-1}+\cdots+a_{n} z^{-n}$

$B\left(z^{-1}\right)=b_{0}+b_{1} z^{-1} \ldots+b_{n} z^{-(n-1)}$

$\xi(t)=0$ (white noise)

Thus,

$$
\begin{aligned}
& A\left(z^{-1}\right) y(t)=B\left(z^{-1}\right) w(t) \\
& y(t)+a_{1} y(t-1)+\cdots+a_{n} y\left(t-n_{1}\right)=b_{0} u(t)+ \\
& b_{1} u(t-1)+\cdots+b_{n} u(t-(n-1))
\end{aligned}
$$

After selection of the model structure, the parameters and system's behavior are predicted using RLS and ANFIS.

\section{B. Recursive Least Square}

For recursive, we consider the effect of additional observation data $\left(\mathrm{Y}_{\mathrm{N}+1}\right)$ as compared with least square estimation [13].

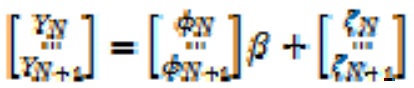

Rearrange in one row of matrix:

$$
\begin{aligned}
& X_{N+1}^{T}=[-y(N) \ldots-y(N)+1-n) w(N) \ldots w(N+ \\
& 1-n)]
\end{aligned}
$$

Then, $\beta$ formula from least square is used together with the effect of new data.

$$
\beta=\left(\varphi^{T} \varphi\right)^{-1} \phi^{T} y
$$

In simplified formulation:

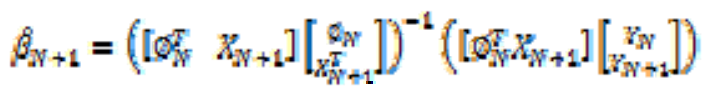

$$
\beta_{N+1}=\hat{\beta}_{W}+K_{N+1}\left[Y_{N+1}-X_{W+1}^{T} \hat{\beta}_{N}\right]
$$

where:

$$
\begin{aligned}
& K_{N+1}=\frac{R_{N} X_{W+1}}{\lambda+X_{W+1}^{T} R_{N} X_{W+1}} \\
& R_{N+1}=\frac{F_{W}}{\lambda}\left(I-\frac{X_{W+1} X_{W+1} R_{N}}{\lambda+X_{W+1}^{T} R_{N} X_{N+1}}\right)
\end{aligned}
$$

\section{Neuro-Fuzzy Modeling}

Adaptive network based fuzzy inference system (ANFIS) is the fuzzy inference system that is built with the structure of adaptive networks as shown in Fig. 4. Adaptive network (AN) as the name suggested is the neural networks structure consisting of nodes and directional links which apply the supervised learning capability. The output will be affected by the parameters from the nodes while the learning rule will determine the behaviour of the parameter to minimize the error. Fuzzy 
inference system (FIS) encompasses of five main functional blocks [14]:

- Rule base - has a number of fuzzy if-then rules

- Database - defines the membership functions

- Decision making unit - performs the inference operations on the rules

- Fuzzification interface - transforms the crisp inputs into degree of match with linguistic values

- Defuzzification interface - converts the fuzzy results into a crisp output

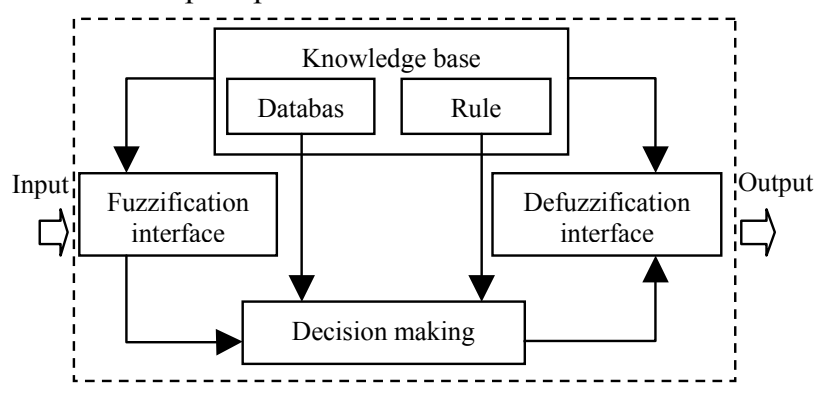

Figure 4. Fuzzy inference system [14]

The ANFIS architecture applied in this paper is the Takagi-Sugeno type of neurofuzzy model (Fig. 5) since it is based on the linear combination of the input and output.

Layer 1: Each neuron $I$ is adaptive with parametric activation function. Its output is the grade of membership to which the given input satisfies the membership function. Parameters in that layer are called premise parameters.

Layer 2: Every node is a fixed node and the output is the product of all incoming signals. The node output represents the firing strength, $\alpha_{\mathrm{i}}$ of the $i$ th rule.

Layer 3: Each node is a fixed node that calculates the ratio of the $i$ th rule's firing strength relative to the sum of all rule's firing strengths. The result is a normalized firing strength.

$\bar{\kappa}_{l}=\frac{\kappa_{l}}{\kappa_{1}+\alpha_{2}^{2}}, i=1,2$

Layer 4: The nodes in layer 4 are adaptive nodes with nodes output of;

$\bar{\alpha}_{0 q}=\bar{\kappa}_{1}\left(c_{11} u_{1}+c_{n_{2}} u_{2}+c_{0}\right), \quad t=1,2$

where

$\bar{\alpha}_{i}=$ normalized firing strength

$\left(c_{\mathrm{T}}, c_{\mathrm{T}}, c_{\mathrm{t}}\right)=$ consequent parameters
Layer 5: Every node is a fixed node which sums all incoming signals.

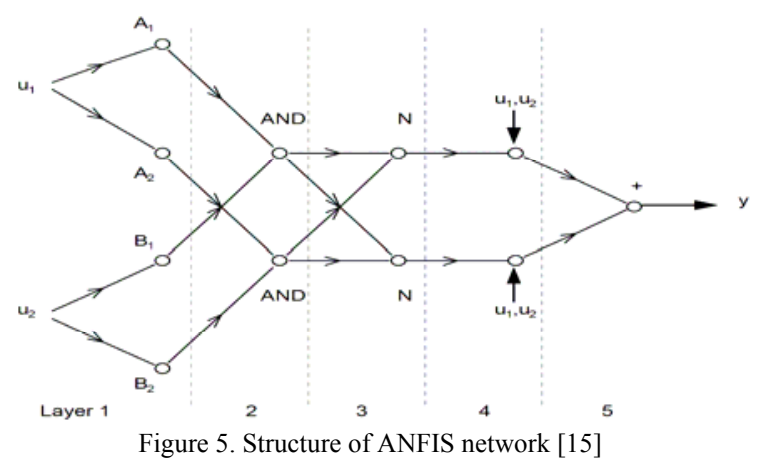

D. Validation Test

It is important to make sure the quality of the model represents the real behavior of the system within the acceptable bounds. This project used the statistical tests on the prediction error in investigating the validity of the model.

\section{Mean Square Error}

Mean squared error is the common variable used in the validation test. MSE is the difference between actual output, y (t), of the system and the predicted output, $f(t)$.

$$
M S E=\frac{1}{X} \sum_{t=1}^{X} y(t)-\varphi(t)^{2}
$$

\section{Correlation Test}

Analyzing autocorrelation and cross-correlation of the residuals with the inputs will be able to give a good indicator in validating a model. These tests provide the measure of how the two variables affect one another. The autocorrelation plot shows the correlation of residuals for each output while cross-correlation plots examine the input and residuals for each input-output pair.

The confidence interval that defines the range of residual values within a specific probability of being statistically insignificant for the system is set at $95 \%$. The time difference between the signals or known as lag is set at -100 to 100 . If the model structure and estimated parameters are correct, then prediction error sequence, $e(t)$, should be uncorrelated with all combinations of past inputs and outputs and this will hold if and only if the following conditions are satisfied [13]:

$$
\begin{aligned}
& \phi_{e q}(\tau) \quad=E[\theta(t-\tau) \theta(t)=\delta(t) \\
& Q_{w \varepsilon}(\tau) \quad=E\left[u(t-\tau) \in(t)=0, \quad \nabla_{t}\right. \\
& \phi_{u} e^{2}(\tau)=E\left[w^{2}(t-\tau)-w^{2}(t) \epsilon(t)=0, \quad z_{\tau}\right. \\
& \phi_{u^{2} s^{2}(\tau)}=E\left[w^{2}(t-\tau)-w^{2}(t) \theta^{2}(t)=0, \psi_{\tau}\right. \\
& \varphi_{(e) \operatorname{lew}(\tau)}(\tau) E[\theta(t) \in(t-1-\tau) w(t-1-\tau)=0 \text {. }
\end{aligned}
$$




$$
\tau \geq 0
$$

where

$$
\begin{aligned}
\Phi_{w \varepsilon}(\tau)= & \text { cross-correlation function between } u(t) \text { and } \\
& e(t) \\
\theta u(t)= & \theta(t+1) u(t+1) \\
\delta \quad= & \text { impulse function }
\end{aligned}
$$
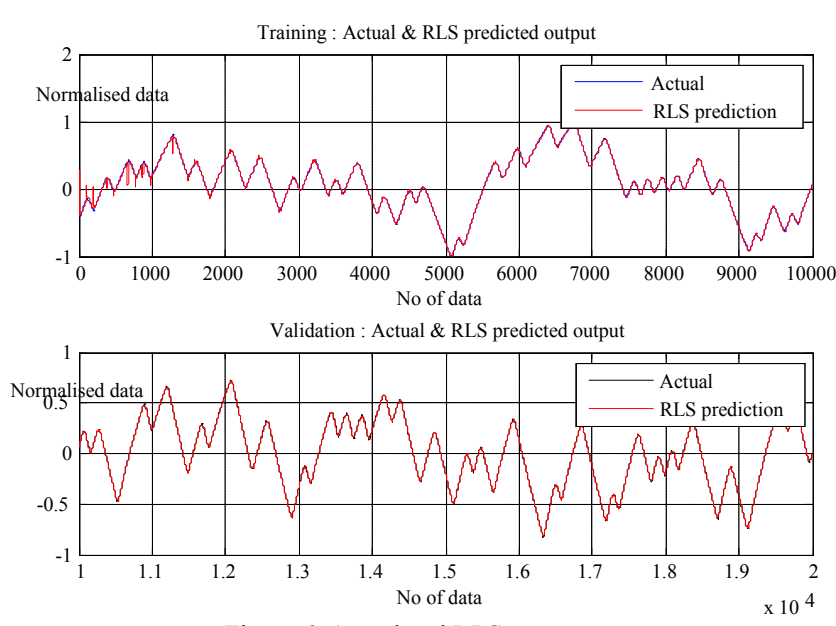

Figure 6. Actual and RLS output

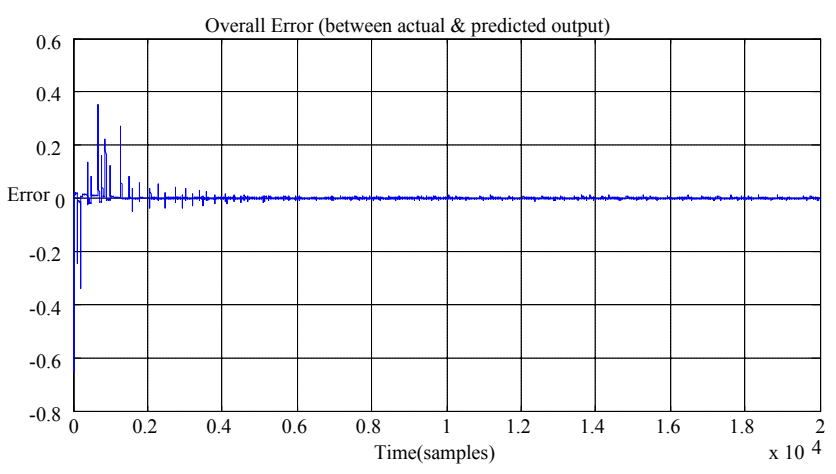

Figure 7. Actual and RLS output

\section{RESULTS \& DISCUSSION}

In finding the best model for RLS, variation of model order and forgetting factor are made to test which one best represents the real system. Mean square error (MSE) and correlation tests are used to validate the model.

\section{A. RLS - Variation of Model Order}

The RLS prediction results by variation of model order with constant forgetting factor, $\lambda=0.9$ is shown in Table 1. From Table 1, the best result is achieved with the model order, $n=2$. Referring to the mean square error, the best MSE value between actual and RLS predicted output of validation data is $9.3545 \mathrm{e}-006$. The data is simulated increasing the model order until model order of 10 and it could be seen that the MSE values also increased.

TABLE I. RLS WITH DIFFERENT MODEL ORDER

\begin{tabular}{ccc}
\hline Model order, $n$ & MSE Training & MSE Validation \\
\hline 2 & $4.0776 \times 10^{4}$ & $9.3545 \times 10^{6}$ \\
3 & $3.9031 \times 10^{4}$ & $1.0124 \times 10^{5}$ \\
4 & $4.7213 \times 10^{4}$ & $1.5373 \times 10^{5}$ \\
5 & $5.0568 \times 10^{4}$ & $2.0146 \times 10^{5}$ \\
10 & $5.5440 \times 10^{4}$ & $4.5028 \times 10^{5}$ \\
\hline
\end{tabular}

\section{B. RLS - Variation of Forgetting Factor}

The RLS prediction results by variation of forgetting factorwith constant model order, $n=2$ is shown in Table 2. From Table II, the best result of MSE is achieved with the forgetting factor, $\lambda$ of 0.9 . This shows that with the high forgetting factor, the contribution of previous data gives big influence to the next predicted output. The larger forgetting factor, $\lambda$ is the larger contribution of previous data. Regression finds the line that best predicts $\mathrm{Y}$ from value X. So, when the value is 1.000 , the estimated data is agreeable with the actual data.

\begin{tabular}{cccc}
\multicolumn{4}{c}{ TABLE II. RLS WITH DIFFERENT FORGETTING FACTOR } \\
\hline$\lambda$ & MSE Training & $\begin{array}{c}\text { MSE } \\
\text { Validation }\end{array}$ & $\begin{array}{c}\text { Regression } \\
(R)\end{array}$ \\
\hline 0.7 & $2.0175 \times 10^{4}$ & $1.0310 \times 10^{5}$ & 1.0000 \\
0.8 & $2.5779 \times 10^{4}$ & $9.8129 \times 10^{6}$ & 1.0000 \\
0.9 & $4.0776 \times 10^{4}$ & $9.3545 \times 10^{6}$ & 1.0000 \\
0.95 & $6.7917 \times 10^{4}$ & $9.6887 \times 10^{6}$ & 1.0000 \\
0.98 & 0.0014 & $1.7153 \times 10^{5}$ & 0.9999 \\
\hline
\end{tabular}

Hence, from both variation of model order and forgetting factor, the best parameters from RLS identification is at model order, $n=2$ with forgetting factor, $\lambda=0.9$. The parameters are $a_{1}=0.5011, a_{2}=$ $0.4977, b_{0}=0.0015$ and $b_{1}=0.0028$.

The correlation tests (Fig. 8) have shown that the residuals or errors of the data are reasonably well uncorrelated with the input and among themselves although not perfectly. When there is a peak outside the confidence interval $(95 \%)$ for lag $k$, the contribution to the output $y(t)$ that originates from the input $u(t-k)$ is not properly described by the model. However, the minor 
fluctuations within the confidence interval are considered to be insignificant. The model thus can be considered satisfied and good. From the validation tests performed, it is concluded that the linear model is sufficient to accurately describe the system dynamics. There is no need to go for complicated or nonlinear model.


Figure 8.RLS prediction correlation tests

Therefore the equation of corresponding model is:

$y(t)=\frac{-(-0.5011) y(t-1)-(-0.4977) y(t-2)+}{0.0015 x(t)+0.0028}+$

In ARX form:

$$
\frac{y(t)}{u(t)}=\frac{0.0015+0.0028 z^{-1}}{1-0.501 .1 z^{-1}-0.4977 z^{-2}}
$$

\section{ANFIS Prediction}

From the result, the ANFIS used here contains 8 fuzzy rules; with total number of fitting parameters are 50 which involved 32 linear parameters and 18 nonlinear parameters. The output graph and error values are referred to Fig. 9 and Fig. 10. The correlation tests of ANFIS prediction have shown that the errors of the data are reasonably well uncorrelated with the input and among themselves within $95 \%$ confidence interval.

Based on the ANFIS model structure (Fig. 11), it could be observed that two membership functions are assigned to each input variable and it contains eight rules. 10000 training data was used to train ANFIS and 10000 validation data were sampled to verify the identified ANFIS only.
The correlation tests for ANFIS prediction (Fig. 12) have shown that the residuals or errors of the data are reasonably well uncorrelated with the input and among themselves.

As the output from recursive least squares (RLS) and adaptive-network-based fuzzy inference system (ANFIS) were compared, the best MSE for RLS is $9.3545 \times 10^{6}$ at model order, $\mathrm{n}=2$, and forgetting factor, $\lambda=0.9$. While for ANFIS, the MSE value is $6.1082 \times 10^{6}$ as shown in Table 3 for the checking data. This shows that the ANFIS has a slightly better performance compared to RLS.
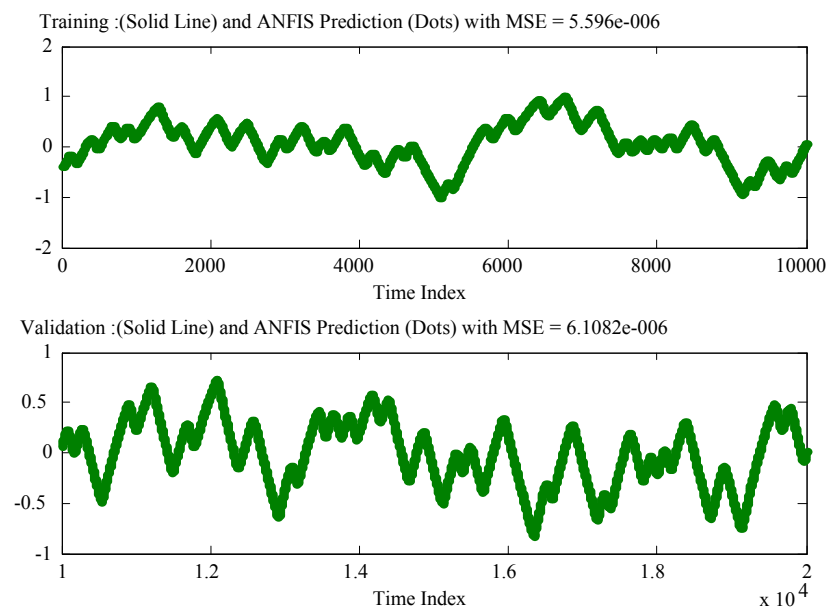

Figure 9. ANFIS output

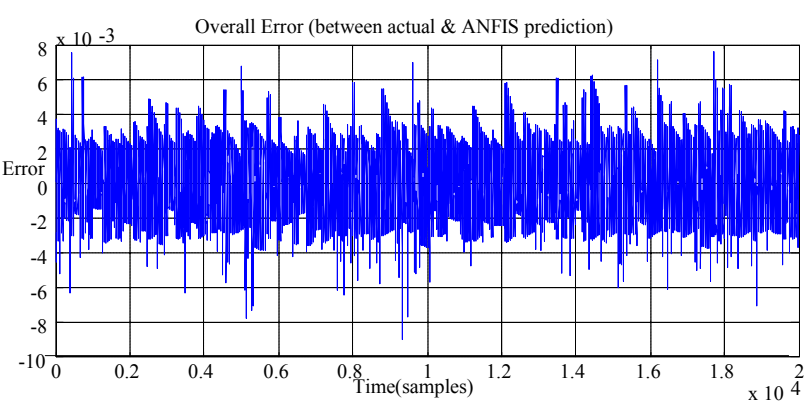

Figure 10. Error between actual and ANFIS prediction

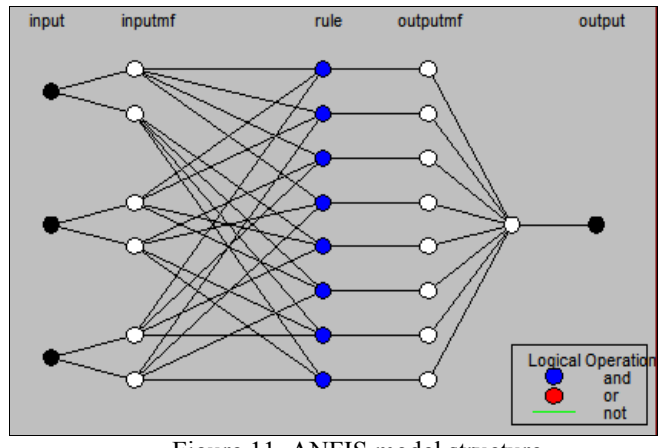

Figure 11. ANFIS model structure 
ANFIS info:

Number of nodes: 34

Number of linear parameters: 32

Number of nonlinear parameters: 18

Total number of parameters: 50

Number of training data pairs: 10000

Number of checking data pairs: 10000

Number of fuzzy rules: 8

TABLE 3. ANFIS PREDICTION

\begin{tabular}{cccccc}
\hline \multicolumn{2}{c}{$\begin{array}{c}\text { Model } \\
\text { Order }\end{array}$} & & $\begin{array}{c}\text { MSE } \\
\text { (Training) }\end{array}$ & $\begin{array}{c}\text { MSE } \\
\text { (Validation) }\end{array}$ & $\begin{array}{c}\text { Regression } \\
(\boldsymbol{R})\end{array}$ \\
\cline { 1 - 2 }$n_{a}$ & $n_{b}$ & $d$ & & & \\
\hline 9 & 10 & 1 & $5.596 \times 10^{6}$ & $6.1082 \times 10^{6}$ & 1.0000 \\
\hline
\end{tabular}

The correlation tests (Fig. 12) have shown that the residuals or errors of the data are reasonably well uncorrelated with the input and among themselves.

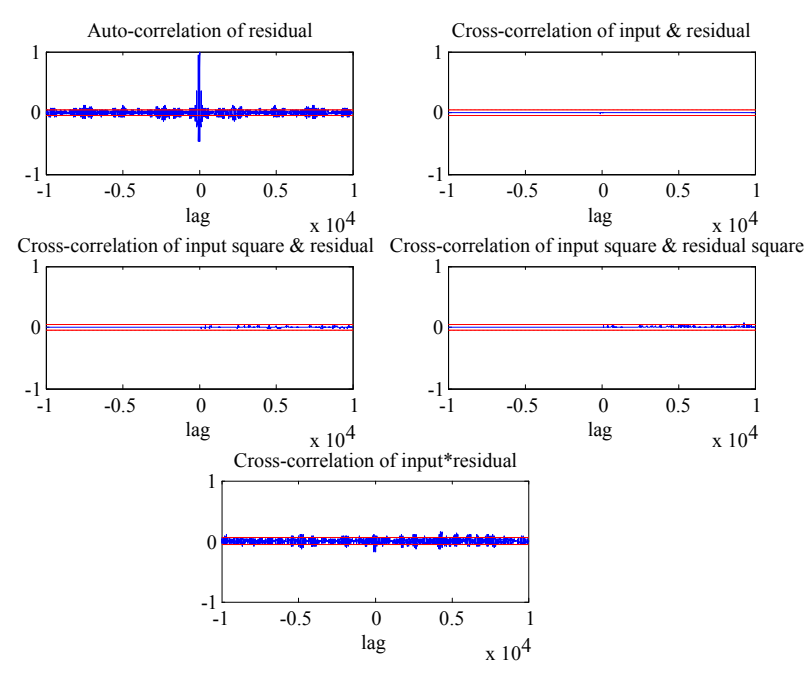

Figure 12. ANFIS prediction correlation tests

\section{CONCLUSION \& FUTURE WORK}

The engine modeling via identification by recursive least squares (RLS) and adaptive-network-based fuzzy inference system (ANFIS) have shown good results. Although ANFIS has smaller MSE value than RLS, but both methods either parametric or non-parametric proved that they can forecast the system behavior well with a very small error.

Further work will involve the controller design in order to resolve some issues related to the ICE as idle speed control and air-fuel-ratio (AFR) control. Other intelligent methods such as genetic algorithm (GA) and neuralnetwork $(\mathrm{NN})$ could be applied to compare the performance among them.

\section{ACKNOWLEDGMENT}

The authors wish to thank the Ministry of Higher Education (MOHE), Universiti Teknologi Malaysia (UTM) and International Islamic University Malaysia (IIUM) for providing the research grant and facilities. This research is supported using UTM Research University grant, Vote No. 04H17.

\section{REFERENCES}

[1] N. Togun, S. Baysec, and T. Kara, "Nonlinear modelling and identification of a spark ignition engine torque", in Mechanical Systems and Signal Processing, 2011.

[2] O.H.N. Hamad, "System identification and design PID controller for automotive air conditioning system", in Faculty of Mechanical Engineering. 2011, Universiti Teknologi Malaysia: Johor.

[3] A.Z.M. Sofi, "Parametric system identification and active vibration control of vibrational structures using genetic algorithm", in Faculty of Mechanical Engineering. 2007, Universiti Teknologi Malaysia: Johor.

[4] S. Haykin, Adaptive filter theory. 2 ed. 1991, Englewood Cliffs, NJ: Prentice-Hall.

[5] T. C. Lee, et. al., "A composition estimator for multicomponent flash drum using recursive equation error method", in Computers and Chemical Engineering, 2000. 24: p. 1281-1286.

[6] S.-M. Moon, R.L. Clark, and D.G. Cole, "The recursive generalized predictive feedback control: theory and experiments",in Journal of Sound and Vibration, 2005. 279: p. 171-199.

[7] C. Bazin and M. Franklin, "Real-time material balance for flotation plants using a least-squares recursive algorithm", in International Journal of Mineral Processing, 1996. 46: p. 231244.

[8] F. Gustafsson, Adaptive filtering and change detection. 2000, England: John Wiley \& Sons Ltd.

[9] K.P. Mohanadas and S. Karimulla, "Fuzzy and neuro-fuzzy modelling and control of nonlinear systems".

[10] K. Butts, "The simulink model", in Ford Motor Company, M.b.P. Barnard, T. Liefeld, and S. Quinn, Editors, Mathworks (1994-1997).

[11] P.R. Crossley and J.A. Cook. "A nonlinear engine model for drivetrain system development", in International Conference on Control 1991. Edinburgh, UK.

[12] R.G.K.M. Aarts, "System identification and parameter estimation". 1998, Enschede: University Twente.

[13] A. R. Tavakolpour, I. Z. Mat Darus, M. Mailah, M.O. Tokhi, "Genetic Algorithm-based Identification of a Rectangular Flexible Plate System for Active Vibration Control", 
T N A TUAN KAMARUDDIN et al: NEURO-FUZZY IDENTIFICATION OF AN INTERNAL COMBUSTION ENGINE

International Journal of Engineering Application of Artificial Intelligence, 2010, 23, pp. 1388 - 1397.

[14] J.-S.R. Jang, “ANFIS: Adaptive-network-based fuzzy inference system", in IEEE Transactions on Systems, Man, and Cybernetics, 1993. 23(3).
[15] J. Jantzen, "Neurofuzzy modelling". 1998, Technical University of Denmark: Denmark. 